\title{
QoS Measurement-Based CAC for an IP Telephony System
}

\author{
José M Maldaña, José I. Aznar, Eduardo Viruete, Julián Fernández-Navajas, \\ and José Ruiz \\ Communication Technologies Group (GTC) - Aragon Institute of Engineering Research (I3A) \\ Dpt. IEC. Ada Byron Building. CPS Univ. Zaragoza \\ 50018 Zaragoza, Spain \\ Tel.: +34976762698 \\ \{jsaldana, jiaznar, eviruete, navajas, jruiz\}@unizar.es
}

\begin{abstract}
This work presents a Call Admission Control (CAC) system for a SIP-based IP Telephony platform. Configured for a multi-branch enterprise environment, the system enables international calls to be established in two steps: one step using Voice over IP (VoIP) through the Internet between the local office and a VoIP-PSTN gateway placed at destination country, and a second step by means of PSTN, from the gateway to the end-user, accounted with local tariffs. CAC decisions are based on Quality of Service (QoS) measurements, call tariffs and also on the number of available lines in the gateway. The CAC has been implemented within a test platform based on virtualization. Measurements to evaluate and validate CAC's impairment on call establishment delays have been obtained.
\end{abstract}

Keywords: IP Telephony, VoIP, CAC, MBAC, SIP, virtualization, QoS.

\section{Introduction}

The use of Internet for voice communications in corporate environments may entail cost savings for enterprises. Conference calls among offices can be carried out using Voice over IP (VoIP) services. For enterprises with offices in several countries, a significant enhancement on communications consists of making use of VoIP services for international conference calls between traditional end-user terminals. These calls can be delivered in two differentiated steps: one step through the Internet by means of VoIP between the enterprise offices, and a second step through the PSTN to reach the end user and accounted with local call tariffs (Fig. 1b).

More specifically, the use of IP Telephony [1] services represents an interesting solution for enterprises since not only implies savings, but also availability and security features.

Users demand a Quality of Service (QoS) similar to the one guaranteed for PSTN. VoIP is a real time service in which the packet delay parameter directly impairs calls' quality. The maximum One Way Delay (OWD) recommended by ITU is $150 \mathrm{~ms}$. [2]. Regarding other QoS parameters, nowadays there exist several solutions for their enhancement related to both control and data planes [3]. At the control plane, the

N. Bartolini et al. (Eds.): QShine/AAA-IDEA 2009, LNICST 22, pp. 3-19, 2009.

(C) Institute for Computer Science, Social-Informatics and Telecommunications Engineering 2009 


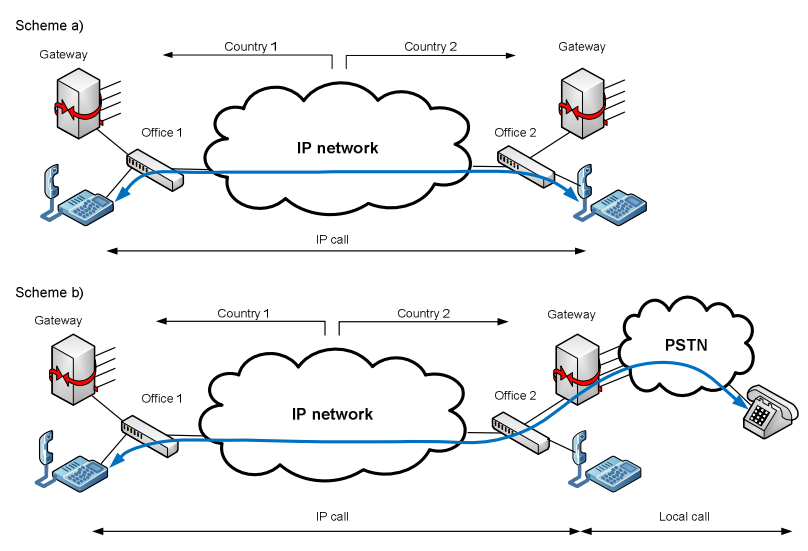

Fig. 1. Traditional and proposed scheme

so-called Call Admission Control (CAC) solution accepts or rejects calls depending on resource availability.

A possible improvement for CAC systems (in terms of QoS and expenses), consists of best route discovery mechanisms for call establishment taking into account that several available locations, from where the call could be established, may exist. To this target, MBAC (Measurement-Based CAC) [4] systems represent a smart option. This work presents a QoS Measurement-Based CAC system developed for a situation in which users have neither control over the Internet, nor over network parameters and the infrastructure. An End-to-End estimation system carries out the measurement of the most relevant QoS parameters over the scenario.

Before setting up an IP Telephony system over the Internet, it is highly recommended to first validate the solution in a controlled environment, such as a testbed platform where several CAC alternatives can be set up and evaluated. Virtualization-based platforms allow deploying a complete network scenario within a single physical machine. Besides, multimedia applications and real protocols can be implemented, achieving realism with low performance cost.

This paper is organized as follows: section 2 discusses the QoS related problems, and their solution using CAC. Section 3 presents the security considerations of this work. System architecture is presented in section 4 . The next section covers the test platform. Section 6 presents the validation and preliminary measurements carried out. The last section details the conclusions of the present work.

\section{QOS Problem: CAC}

Concerning VoIP sphere, there exists a large variety of protocols and configurations for both multimedia data and signaling. The Real Time Protocol (RTP) is usually used for multimedia transmission. At signaling plane, SIP (Session Initiation Protocol) [5], H.323, IAX (Inter-Asterisk eXchange) or MGCP (Media Gateway Control Protocol) are some of the adopted standards. The analysis performed in this work is SIP-based, since it is and open protocol that is widely used in IP networks [6]. 
Up to now, a complete solution for providing signaling protocols with dynamic configuration and management of QoS parameters does not exist. QoS-guaranteed networks are not prepared to support the massive implementation of multimedia services. Integrated Services (IntServ) [7] mechanisms require all routers along the path to cache signaling information related to each flows, and represents a non scalable solution. On the other hand, Differentiated Services (DiffServ) [8, 9] make use of the Type of Service (ToS) field for traffic classification and also set up a collection of generic rules which establish how each node should react to each traffic flow (Per-Hop Behaviour, PHB). Besides, main problems of this architecture lie in the fact that a mapping process between applications and service classes is first required, and normalising RFC documents still have many open doors to its implementation.

As we previously commented, CAC [10] systems improve call's QoS: They accept or reject new incoming calls depending on the network behaviour. New incoming call acceptance paradigm consists of, while accepting the call, the remaining ongoing calls are not affected in terms of quality, packet losses and delays [11].

A variety of CAC systems have been widely used over several network technologies, such as mobile networks or ATM [12]. Today, in fact, CAC systems constitute a key component in QoS networks defined by NGN (Next Generation network) standardization organisms like 3GPP (3rd Generation Partnership Project), WiMAX Forum (Worldwide Interoperability for Microwave Access) and TISPAN (Telecommunications and Internet converged Services and Protocols for Advanced Networking). Recommendations define a central entity for the management of QoS policies and resources supply. In this scenario, SIP has been adopted by 3GPP as IMS (IP Multimedia Subsystem) [13] signaling protocol.

MBAC represents a suitable CAC option for QoS enhancement. Nowadays, these systems are used in some commercial solutions [14] but their features are limited to manufacturer's devices. For instance, Cisco presents two MBAC SIP compatible systems: AVBO and PSTN Callback [15]. Some other systems are [11]: SU-CAC (Site Utilization-Based CAC), which reserves bandwidth for VoIP communications in the configuration stage, and LU-CAC (Link Utilization-Based CAC), whose decisions are based on host individual bandwidth usage, enabling layer-2 multiplexing, but increasing complexity to the system and using RSVP (Resource ReSerVation Protocol) [16].

The following conditions $[12,17]$ need to be satisfied for a functional MBAC scheme:

- $\quad$ Ensure that desired QoS level is targeted (precision).

- Maximize resources usage.

- Reach a tradeoff between implementation expenses and revenues.

Recently, different MBAC designs for real time flows, voice and video essentially, have been developed. In [18] it is presented a CAC system that aims to preserve QoS parameters in a wireless mesh network, for a VoIP service. Reference [19] presents a predictive autoregressive CAC algorithm for video distribution systems.

Since MBAC is based on measurements, its implementation requires the usage of estimation and monitoring tools for QoS parameters. There exist several tools (e.g. Nettimer, Pathchar, etc.) which characterize delay, jitter delay, available bandwidth and losses. Depending on the tool, several MBAC systems can be configured. These 
tools can be classified in two groups: End-to-End and centralized. The first ones are used to measure parameters at network borders, without considering the inner network structure. On the other hand, centralized tools make use of information obtained from routers' statistics. In case the MBAC system has no control over the network, End-to-End measurement tools are adopted.

Likewise, another classification for QoS measurement tools divide them in active [20] and passive [21] categories. Active tools are based on analyzing the so-called probe packets, delivered into the network. Passive ones, capture packets corresponding to network flows for their analysis either online or offline.

\section{Security Considerations}

One first option to secure users' flows consists of using IPsec at IP level. In fact, enterprises tend to adopt VPN systems for communications among offices. The problem of this solution is that it demands from the VPN-gateways installed in the data centre and offices to be fast enough not to add undesirable delays in voice packets.

Security can also be managed at transport layer. SIP over Transport Layer Security (TLS) protocol is called SIPS. This protocol ensures hop-by-hop security, so that it might only be interesting in case all SIP entities have wholesale relationships and they use PKI (Public Key Infrastructure) for authentication processes. Besides, TLS runs over TCP, while the most suitable protocol for real time traffic is UDP.

Security can also be configured within SIP messages at application layer, as described in RFC 3261: S/MIME authenticates and encodes users' messages. Digest authentication is an accepted method too.

To secure multimedia traffic, SRTP can be used. This protocol provides confidentiality, integrity and authentication to the system and hardly impairs the frame size. AES (Advanced Encryption Standard) is the encoding algorithm for multimedia content. It enables individual frame decoding, a basic feature for real time flows where losses and out of order packet delivery are common.

\section{System Architecture}

\subsection{General Scenario Description}

In this article, we implement an End-to-End based MBAC for an IP Telephony system. The initial scheme is similar to the one implemented by Cisco [11]. The IP Telephony system network corresponds to an enterprise with several central offices placed in different countries (Fig. 2). Each office has its own local agent and the PBX is configured within the centralized data centre. To reduce management expenses, it is desirable to keep the dialplan only for the PBX and not to distribute it to the central offices. Furthermore, Internet is used for Telephone traffic delivery among offices, instead of dedicated lines. For a suitable system performance, each office also includes IP-telephones, soft phones, and a VoIP gateway. 


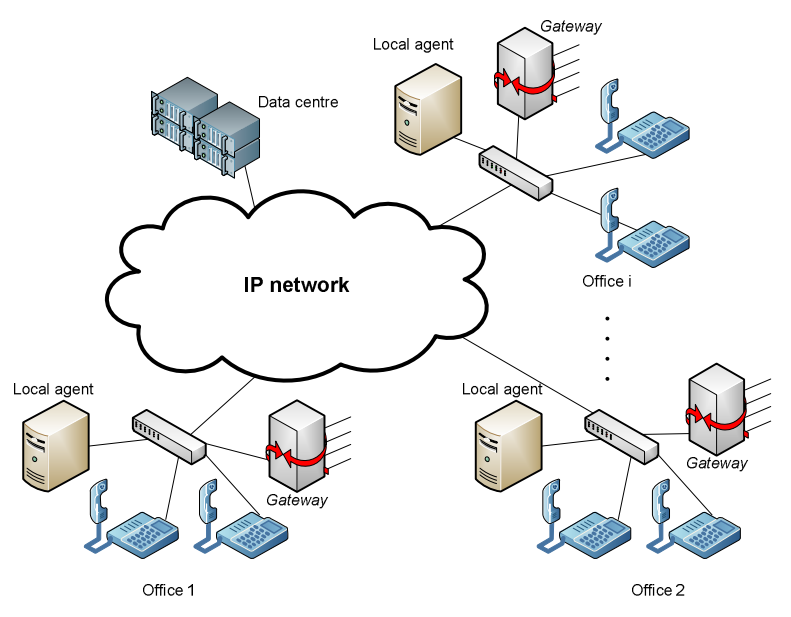

Fig. 2. System architecture

Local agents play a fundamental role in choosing the best route for phone calls in terms of QoS and expenses. First, estimation and monitoring processes of QoS parameters carry out measurements among offices, collecting relevant information for the system. Taking on account this information, the phone tariffs and the number of available and occupied lines in the gateway, the local agent fills in the tables on which the MBAC will base its decisions. Finally, the agent, through its SIP proxy, processes the phone calls signaling to implement the CAC mechanism, based on the information provided by the tables.

This work relies on the following assumptions:

1) The measurement system has been designed to dynamically adapt to connection characteristics with a tight inter-estimation time. A high inter-estimation time would derive a lack of accuracy in the measurements, and a short one may result in network overloading.

2) The data centre comprises a high availability system, security, back-up files and other functionalities to ensure a proper operational behavior without interruptions. A broadband Internet connection with enough bandwidth is also available.

3) There exists a function that calculates and takes the CAC decision based on QoS measurements, accounting tariffs and lines' occupancy in gateways.

\subsection{CAC System Operation}

As it has been depicted, the system includes a single PBX that includes the dial plan. There is a local agent in each central-office in charge of signaling, configured in such a way that all signaling messages among the PBX and the terminals will pass through it. Thus, signaling information can be sent to the CAC in order to take decisions about future connection requests and keep count of the number of calls established in the 
gateway at any time. In case no call rejections are notified, the agent only retransmits signaling messages.

Internal office calls are directly managed by the local agent and do not require PBX functions to be established. For cases in which calls between offices do not go through the PSTN, CAC decisions are just for call acceptance or rejection, since it would be worthless to redirect them to an office different from the destination one.

In order to adopt CAC decisions, each local agent makes use of a so-called "decision table" (Table 1), in which it is specified how the agent may act in case a call request (INVITE) SIP message is received.

Table 1. Decision table

\begin{tabular}{|l|l|l|}
\hline Origin & Internal call & call to PSTN \\
\hline 1 & Accept / reject & Accept / reject / redirect to $i$ \\
\hline 2 & Accept / reject & Accept / reject / redirect to $i$ \\
\hline$\cdots$ & $\cdots$ & $\cdots$ \\
\hline N & Accept / reject & Accept / reject / redirect to $i$ \\
\hline
\end{tabular}

This table is built from other tables discussed in next sections, which depend on QoS parameter estimations, telephone tariffs and number of available lines in the gateway.

$$
\text { Decision table }=f(\text { QoS measures, tariffs, available lines })
$$

When a local agent receives an INVITE from the PBX (Fig. 3), sent to a terminal, it accepts or rejects it, depending on the corresponding record filled in the table. The incoming call can be rejected due to lack of QoS in the route between source and destination offices, or due to unavailability of destination terminal.

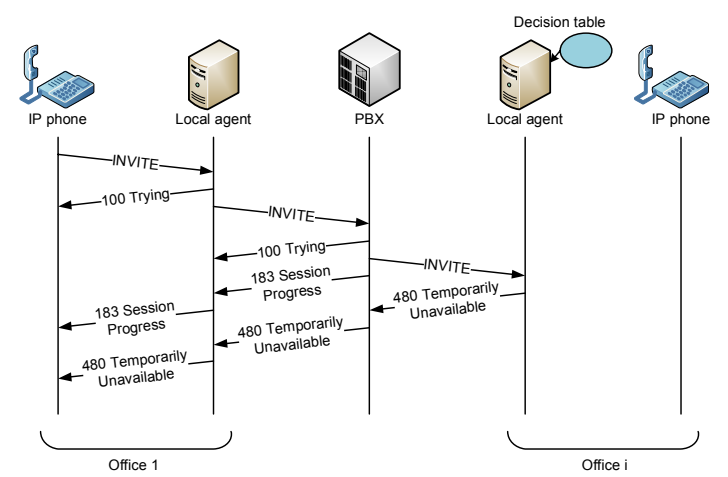

Fig. 3. Rejected call 


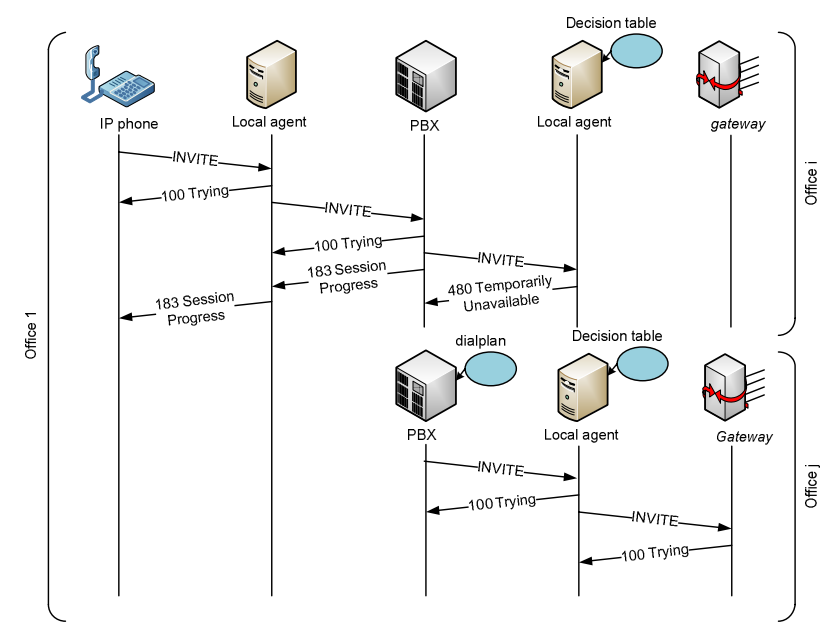

Fig. 4. PBX tries to establish a call by the gateway of another office

In case the local agent receives an INVITE message sent to the gateway and the table indicates that it must be rejected, a "480 Temporarily Unavailable" SIP message (Fig. 4) will be sent to the PBX. The PBX, according to its dial plan, may try to establish the call through other office's gateway with an economic tariff for the destination of the call.

In case the table indicates "redirect", the agent acts as redirect server, re-routing the call to the central office which owns available lines to establish the connection (Fig 5). The redirect server sends a $3 \mathrm{XX}$ message reporting about an alternative route.

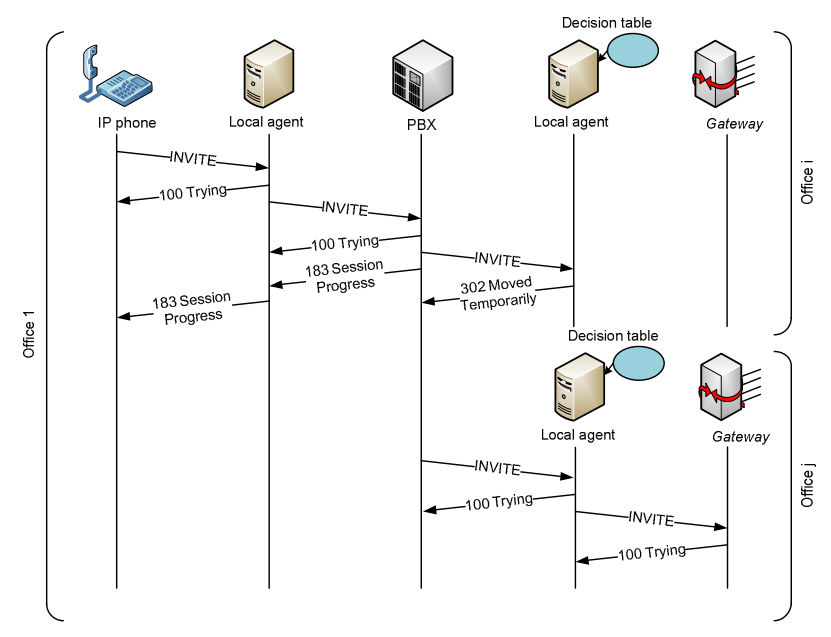

Fig. 5. A call is redirected to a new office 
Then, the PBX tries to reach the new destination without consulting its dial plan. The agent who acts as redirect server will not take part in this call again.

\subsection{Control Tables}

As we have previously indicated, each agent's "decision table" is filled in from other tables that are next described:

1) QoS table (Table 2): In each office, a table containing QoS parameter estimations is configured. This table is not mandatory to be symmetric, since there exist broadband access networks that neither are. Elements belonging to the diagonal are not defined since they would represent measurements inside an office. Depending on the measurements considered, it may exist several tables with different QoS parameters.

Table 2. QoS table

\begin{tabular}{|l|l|l|l|l|}
\hline & Agent 1 & Agent 2 & $\cdots$ & Agent i \\
\hline Agent 1 & - & Par 1 $\rightarrow 2$ & $\cdots$ & Par 1 $\rightarrow \mathrm{i}$ \\
\hline Agent 2 & Par 2 $\rightarrow 1$ & - & $\cdots$ & Par 2 $\rightarrow \mathrm{i}$ \\
\hline$\cdots$ & $\ldots$ & $\cdots$ & $\cdots$ & $\cdots$ \\
\hline Agent i & Par i $\rightarrow 1$ & Par $i \rightarrow 2$ & $\cdots$ & - \\
\hline
\end{tabular}

2) Tariff table (Table 3): Each field is an integer number representing a tariff rate, corresponding to a call from an origin office gateway $i$ to a destination country $j$. Local calls are designed as "level 1" and the level increases as the price does.

Table 3. Tariff table

\begin{tabular}{|l|l|l|l|l|}
\hline & Country 1 & Country 2 & $\cdots$ & Country $\mathbf{j}$ \\
\hline Gateway 1 & Tariff $1 \rightarrow 1$ & Tariff $1 \rightarrow 2$ & $\cdots$ & Tariff $1 \rightarrow j$ \\
\hline Gateway 2 & Tariff $2 \rightarrow 1$ & Tariff $2 \rightarrow 2$ & $\cdots$ & Tariff $2 \rightarrow j$ \\
\hline$\cdots$ & $\cdots$ & $\cdots$ & $\cdots$ & $\cdots$ \\
\hline Gateway $\mathbf{i}$ & Tariff $i \rightarrow 1$ & Tariff $i \rightarrow 2$ & $\cdots$ & Tariff $i \rightarrow j$ \\
\hline
\end{tabular}

3) Gateway line table (Table 4): This table lets the system know PSTN lines available at each office. First column indicates the total number of lines in the gateway. The second one indicates the number of occupied lines. 
Table 4. Gateway line table

\begin{tabular}{|l|l|l|}
\hline & Total lines & Busy lines \\
\hline Gateway 1 & TL 1 & LO 1 \\
\hline Gateway 2 & TL 2 & LO 2 \\
\hline$\cdots$ & $\cdots$ & $\cdots$ \\
\hline Gateway i & TL i & LO i \\
\hline
\end{tabular}

The table of decisions (Table 1) is filled in from QoS, tariffs and gateway line tables by using (1). This table can be set up from the information of a single office or taking on account the measurements obtained from all offices.

In case each local agent only knew about its own office parameters related to the rest of them, Table 2 would result in a single column array. Gateway line table (Table 4) would be minimized to a counter of available free lines within the office itself. Thus, there would not be information exchange (QoS, lines and tariffs) with the remaining offices. The Tariff table could be completely filled in for all offices beforehand, since its refreshing period is large enough (daily or weekly).

In case local agents have the possibility of collecting information concerning all other offices, the function in (1) may have added complexity. If tables' processing time highly increases, (1) might have no validity, in case it runs too long from the instant when measurements were carried out.

The SIP proxy integrated within the agents may act as a redirect server, re-routing connections (Fig. 5) towards the office which presents the best conditions to establish the call. To this target, it is necessary to have information about all other offices; otherwise there could appear undesirable effects (e.g. signaling loops).

\section{Test Platform}

In this chapter we will present the test platform in which the system has been implemented. The design has to adapt well to the IP Telephony system, emulating realistic conditions, and allowing tests and measurements with flexibility.

\subsection{Simulation, Real Environment, or Virtual Emulation}

To build up the platform there are several options to be considered. Simulation tools are one of these options. In fact, they have already been used in other CAC systems studies [22, 23]. There exist several simulation tools (OPNET, OMNET++, NS-2), nevertheless, they do not implement concrete protocols deployed over a real scenario. The testbed platform could be also be implemented with real devices. However, it may result in high hardware costs, due to the large number of devices that make up the scenario. 
Some studies [24, 25] make use of several machines within an only physical computer in order to minimize costs and optimize the testbed control. For instance, User Mode Linux (UML) has been used in the implementation of some emulators like vBET [26]. Virtual nodes connect to each other through an emulated network running under the network card driver. This concept matches with the test environment we want to deploy.

\subsection{Test Platform Requirements}

The most valuable requirement is to achieve a realistic test platform at a low cost. By using virtual machines, we are able to make use of real applications and complete protocol stacks in their most suitable versions.

Regarding scalability, network configuration enables the platform to be extended with physical machines in case computation and calculation needs became higher due to traffic management or the number of virtual nodes taking part in the test.

Additionally, all tests are being carried out within the same physical machine, guaranteeing test repeatability, since we have configured an isolated and controllable platform.

\subsection{Selection of Virtualization Technology}

Virtualization consists of using a set of machines, each of them with its own OS, which are executed over the real hardware of a single physical machine.

Considering all virtualization schemes that can be distinguished [27], we have selected one designed as paravirtualization, which includes within the client OS the required modifications to avoid any instruction to be managed with privileges. This requirement let the environment run with an execution speed close to non virtualized schemes. Besides, several machines can simultaneously run. The solution we have adopted is based on XEN paravirtualization.

Comparative virtualization platform studies [28] have shown XEN as a suitable tool in terms of overhead, linearity and isolation among virtual machines. Communication performance for a scenario composed of 10 virtual machines has been measured in $93 \mathrm{MB} / \mathrm{s}$ between pairs.

These characteristics are highly interesting for the platform performance, since it is desirable to have a controlled environment in which all virtual machines share available resources in an equitable way.

\subsection{Physical Machine Features}

The machine within the platform has been developed works based on CentOS 5 OS. Linux core version is 2.6.18-8.1.15. It has a Core 2 Duo at $2.40 \mathrm{GHz}$ processor, $2 \mathrm{MB}$ of Cache level 2, and 4GB RAM. Virtual machines also work with CentOS 5. The version of Xen is 3.03-25.0.4.

CPU usage has been monitored during the tests, in order to avoid the influence of processor load on measurements. Utilization has never exceeded $10 \%$. 


\subsection{System Implementation in the Test Platform}

The devices chosen for the developed scenario should require low computational load, due to the fact that they run within a virtualized environment. The required components comprise a PBX, soft phones, SIP proxy servers and VoIP gateways. Only free software solutions have been used.

\subsubsection{PBX}

Asterisk 1.6, a software PBX developed by Digium, has been configured. Asterisk represents an interesting solution because of its flexibility, updates and GNU-GPL license distribution. It supports SIP, H.323, IAX and MGCP signaling protocols.

A dial plan has been used to permit the redirection of an incoming call to another office in case the gateway chosen as first option does not accept it.

If a scenario whose PBX can be tuned is considered, it must be taken into account that Asterisk includes the so-called Asterisk RealTtime (ARA) [29] tool, which offers a method to cache and save configuration files in a MySQL or PostgreSQL database. Static mode requires a reload each time a change in the PBX is carried out. On the other hand, the dynamic mode allows Asterisk accessing the database and updating the configuration files in real time. In this case, the dial plan is also dynamically updated according to the QoS parameters measured by agents, gateway availability lines and accounting tariffs.

\subsubsection{SIP Proxy}

Proxy requirements may include redirect server option and the possibility to be tunable so that $\mathrm{CAC}$ decisions can be implemented. It must be capable of accessing external information placed at a database too.

The OpenSIPS 1.4 free software version has been the selected solution. It has register server, location server, proxy server and redirect server functionalities. Low computational load and the possibility to add and delete functionalities in a modular way are also smart features. At transport layer it supports UDP, TCP, TLS and SCTP. At network layer both IPv4 and IPv6 are supported.

SIP proxy configuration is done with a high level programming language, within the opensips.cfg in which it is specified what the proxy should do for each received message. MySQL access is available too.

AAA (Authentication Authorization and Accounting) functions can be managed through databases (MySQL, PostgreSQL or text files), RADIUS or DIAMETER protocols.

\subsubsection{Soft Phone}

PJSUA 1.0 is the soft phone implemented at local offices. It is part of the PJSIP project. This project offers a complete SIP stack under the GPL license. PJSUA is the reference command line soft phone utilized in PJSIP to achieve the whole SIP protocol implementation and its footprint is smaller than 150KB. It supports simultaneous calls, call waiting and voice messages functionalities, UDP, TCP, TLS and SRTP protocols and Speex, iLBC, GSM, G711 and G722 codecs. Finally, there are also available NAT functionalities (ICE and STUN). 


\subsubsection{Gateway}

IP Telephony gateways are also necessary to complete the emulated platform. It must also be taken on account that PSTN connections are also emulated.

The solution adopted makes use of the PJSUA soft phone, since it supports simultaneous calls, this way limiting the number of gateway lines to emulate. Thus, whenever all PJSUA lines are occupied, the system will consider that the gateway has run out of lines and it will reject future calls.

\subsection{System Security}

Since the measurement plan still remains in its early stage, security protocols have not yet been included. We next enumerate considered possibilities.

In case IPsec tunneling was available among the data centre and each office for any traffic flow, it could also be used for voice traffic without requiring security protocols in upper levels. Nevertheless, due to the fact that voice is a real time application, upper layer protocols could in certain situations confer better performance and offer better QoS than IPsec.

SIP signaling can be guaranteed through TLS. The main drawback of this solution lies in the need of having one TLS tunnel per existing link. Besides, TLS works over TCP, and additional delay and overhead are susceptible to impair the system.

Asterisk does not natively support SRTP. Thus, it has been tested to directly send SRTP traffic between two soft phones without going through the PBX. The main issue lies in the fact that the system can only run in this configuration mode when both end user firewalls manage voice traffic. On the other hand, the traffic should also pass through the PBX. In this case TLS should also be used for RTP flows. SIP proxies do not take part in multimedia flow management, since they are only in charge of signaling at the control plane.

Implementing the CAC in the system implies the addition of local agents to the traditional scenario which only includes the PBX and the soft phones. If TLS is the solution adopted, the appropriate certificates must be used for each one of the four TLS tunnels: Between each soft phone and its proxy and between the proxies and the PBX. To configure this TLS scenario, TCP transport for SIP must also be supported within Asterisk. This feature has been already included in Asterisk 1.6. version.

\section{Validation and Preliminary Measures}

The CAC system validation has been carried out over SIP in the test platform, composed of virtual machines with three central offices apart from the PBX. For each test, we have performed 10 measurements to obtain the mean and standard deviation values. These measurements have been performed independently of the transmission and propagation times, since they are not yet implemented in our emulated environment. Thus, we talk about processing time measurements.

We measure $T_{\text {pnoCAC }}$ (machines' processing time without the CAC system) and $T_{p C A C}$ (processing time with CAC). Furthermore, in the emulated scenario, obtained measurements will be impaired due to the fact that all the machines are running over the same hardware; thus, obtained delay measurements represent an upper limit of the 
processing time in real machines. Besides, we define $T_{U L}$ as the propagation delay at the upload link and $T_{D L}$ the propagation delay at the download link (Fig. 6).

Including the local agents entails two new machines in the route of signaling packets to go through. This implies adding two times the delay produced at the local network (Fig. 6 and 7). This delay is negligible compared to Internet propagation delays.

We want to measure the time from the first INVITE to the beginning of the dial tone at destination phone. In a system without CAC this time can be defined as:

$$
T_{n o C A C}=T_{U L}+T_{D L}+T_{\text {pnoCAC }}
$$

In a CAC system, considering negligible the propagation time of the local network, the delay is defined as:

$$
T_{C A C}=T_{U L}+T_{D L}+T_{p C A C}
$$

Table 5 shows measured processing times added by the presence of a CAC system in the scenario.

Table 5. Processing time in ms

\begin{tabular}{|l|l|l|l|}
\hline \multicolumn{2}{|l|}{$T_{\text {pnoCAC }}$} & \multicolumn{2}{l|}{$T_{\text {pCAC }}$} \\
\hline mean & std. dev. & mean & std. dev. \\
\hline 2.36 & 0.52 & 7.87 & 0.83 \\
\hline
\end{tabular}

From (2) and (3), we can obtain the delay that the CAC system introduces as:

$$
T_{C A C}-T_{n o C A C}=T_{p C A C}-T_{p n o C A C}
$$

It can be shown that the CAC system introduces an additional mean delay of $5.5 \mathrm{~ms}$. This time is significantly lower compared to the usual propagation and transmission Internet delays, thus the CAC system may not entail a quality impairment for VoIP communications.

The previous establishment call delays have also been compared to the ones obtained when a call is redirected to another central office, according to the CAC system decision (Table 6). The local agent acts as a redirect server forwarding the call to the PBX, and from there to another central office.

Table 6. Redirect delay in ms

\begin{tabular}{|l|l|}
\hline \multicolumn{2}{|l|}{$T_{\text {Pre }}$} \\
\hline mean & std. dev. \\
\hline 12.84 & 0.72 \\
\hline
\end{tabular}




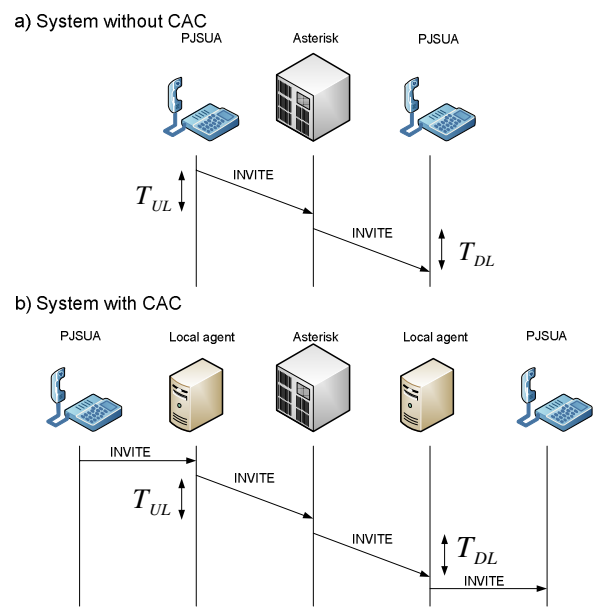

Fig. 6. Call request with and without CAC

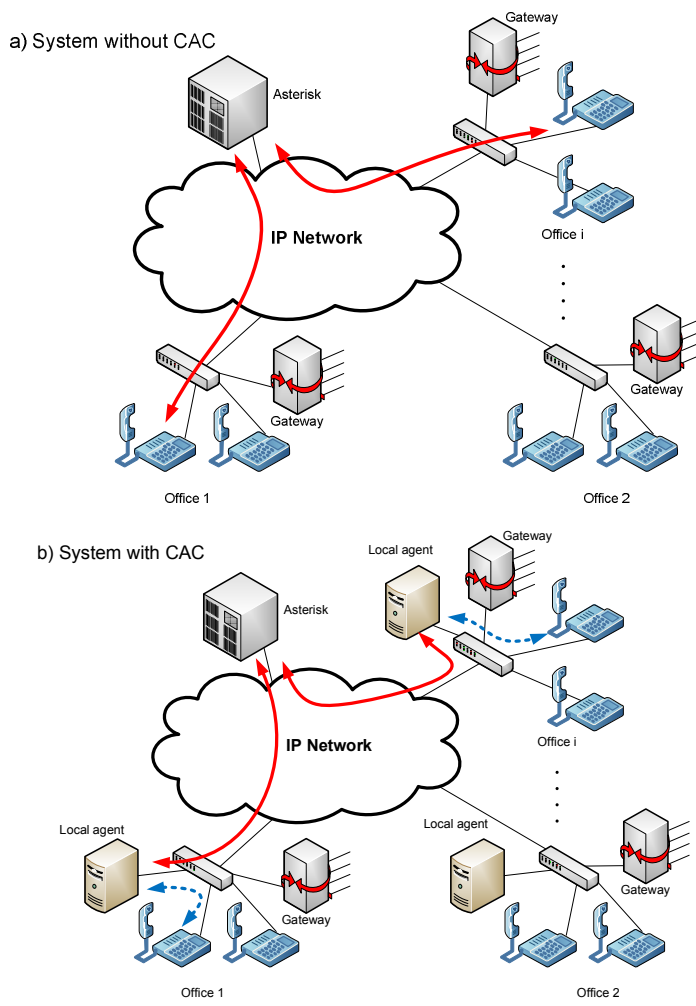

Fig. 7. Comparative of the system with and without CAC 
Redirecting a call entails a total processing delay designed as $T_{\text {Pre }}$. We show that this delay is about $5 \mathrm{~ms}$, that is, the difference between the Table 6 delays and the $T_{P C A C}$ measured in Table 5 .

Besides this additional delay, we must consider the transmission delay that brings up for the call signaling messages in each one of the links between central offices involved in the redirecting process to the PBX. This delay depends on the considered scenario and the number of redirections that a certain call needs. Let $T_{U L i}$ be the uplink delay of the $i$ office, and $T_{D L i}$ the downlink delay. The delay of a call that has been redirected $N$ times is defined as:

$$
T_{\text {redir }}=T_{p C A C}+N \cdot T_{p_{\mathrm{Re}}}+T_{U L_{1}}+T_{D L_{N+2}}+\sum_{i=2}^{N+1}\left(T_{D L_{i}}+T_{U L_{i}}\right)
$$

Depending on each office's delay and the number of redirections carried out, resulting $T_{\text {redir }}$ value might be inadmissible. Thus, it is absolutely unavoidable to obtain this $T_{\text {redir }}$ measurement for each tested environment to ensure a suitable and proper behaviour of the CAC system.

\section{Conclusions}

In this article, we have presented a proof of concept of a CAC system for SIP-based IP Telephony platforms, based on QoS measurements. According to these measurements, the number of lines available at the gateway and the accounting tariffs, accepts, rejects or redirects the call towards another central office. The tables on which CAC decisions are based, have been also discussed.

We have implemented a test platform composed of three central offices, based on a virtualization environment. Suitable software, PBX, SIP proxy, soft phone and gateways complete the emulated platform.

Finally, the system has been properly validated by carrying out processing delay measurements. It has been observed that these delays do not significantly impair the call establishment. Nevertheless, the call establishment delay could be highly impaired in case the number of redirections increases too much.

\section{Acknowledgements}

This work has been partially financed by RUBENS (Rethinking the Use of Broadband access for Experience-optimized Networks and Services) project, of EUREKA CELTIC (code EU-3187 CP5-020) European project, and the project TSI-0204002008-020 of AVANZA I+D sub-programme, of the Spanish Ministry of Industry, Tourism and Commerce.

\section{References}

1. Bearden, M., Denby, L., Karacali, B., Meloche, J., Stott, D.T.: Assessing Network Readiness for IP Telephony. In: Proc. of the 2002 IEEE International Conference on Communications, ICC 2002 (2002) 
2. One-way transmission time (recommendation g.114). International Telecommunication Union (ITU) (February 1996)

3. Chen, X., Wang, C., Xuan, D., Li, Z., Min, Y., Zhao, W.: Survey on QoS Management of VoIP. In: Proc. of the 2003 International Conference on Computer Networks and Mobile Computing. IEEE Computer Society, Los Alamitos (2003)

4. Jiang, Y., Emstad, P.J., Nicola, V., Nevin, A.: Measurement-based admission control: A revisit. In: 17th Nordic Teletraffic Seminar (2004)

5. Rosenberg, J., et al.: SIP: Session initiation Protocol, RFC 3621 (2002)

6. Zave, P.: Understanding SIP through Model-Checking. In: Schulzrinne, H., State, R., Niccolini, S. (eds.) IPTComm 2008. LNCS, vol. 5310, pp. 256-279. Springer, Heidelberg (2008)

7. Braden, R., Clark, D., Shenker, S.: Integrated Services in the Internet Architecture: an Overview. RFC 1633 (1994)

8. Nichols, K., et al.: Definition of the Differentiated Services Field (DS Field) in the IPv4 and IPv6 Headers, RFC 2474 (1998)

9. Blake, S., et al.: An Architecture for Differentiated Services, RFC 2475 (1998)

10. Yu, J., Al-Ajarmeh, I.: Call Admission Control and Traffic Engineering of VoIP. In: Second Intenational Conference on Digital Telecommunications, IEEE ICDT 2007 (2007)

11. Wang, S., Mai, Z., Xuan, D., Zhao, W.: Design and implementation of QoS-provisioning system for voice over IP. IEEE Transactions on Parallel and Distributed Systems 17(3), 276-288 (2006)

12. Mao, G., Habibi, D.: Loss Performance Analysis for Heterogeneous ON-OFF Sources with Application to Connection Admission Control. IEEE/ACM Transactions on Networking (February 2002)

13. 3GPP TS 24.228 v5.15.0, Signalling flows for the IP multimedia call control based on Session Initiation Protocol (SIP) and Session Description Protocol (SDP), Stage 3, R5 (September 2006)

14. SIP: Measurement-Based Call Admission Control for SIP, http://www.cisco.com/en/US/docs/ios/12_2t/12_2t15/feature/ guide/ftcacsip.pdf

15. VoIP Call Admission Control, http://www.cisco.com/en/us/docs/ios/solutions_docs/ voip_solutions/CAC.pdf

16. Braden, R., et al.: Resource ReSerVation Protocol (RSVP), RFC 2205 (1997)

17. Grossglauser, M., Tse, D.: A Time-Scale Decomposition Approach to Measurement-Based Admission Control. IEEE/ACM Transactions on Networking (August 2003)

18. Wei, H., Kim, K., Kashyap, A., Ganguly, S.: On Admission of VoIP Calls Over Wireless Mesh Network. In: IEEE International Conference on ICC 2006, June 2006, vol. 5, pp. 1990-1995 (2006)

19. Camarda, P., Guaragnella, C., Striccoli, D.: A New MBAC Algorithm for Video Streaming Based on Autoregressive Adaptive Filtering. In: IEEE International Conference on Multimedia and Expo., pp. 1512-1515 (2005)

20. Ivars, I.M., Karlsson, G.: PBAC: Probe-Based Admission Control. In: Smirnov, M., Crowcroft, J., Roberts, J., Boavida, F. (eds.) QofIS 2001. LNCS, vol. 2156, pp. 97-109. Springer, Heidelberg (2001)

21. Cetinkaya, C., Knightly, E.: Egress Admission Control. In: Proc. IEEE INFOCOM 2000 (March 2000) 
22. Alipour, E., Mohammadi, K.: Adaptive Admission Control for Quality of Service Guarantee in Differentiated Services Networks.International Journal of Computer Science and Network Security, IJCSNS 8(6) (June 2008)

23. Tran, H.T., Ziegler, T., Ricciato, F.: QoS Provisioning for VoIP Traffic by Deploying Admission Control. In: Burakowski, W., Bęben, A., Koch, B. (eds.) Art-QoS 2003. LNCS, vol. 2698, pp. 1084-1085. Springer, Heidelberg (2003)

24. Zhou, J., Ji, Z., Bagrodia, R.: TWINE: A Hybrid Emulation Testbed for Wireless Networks and Applications. In: Proc. IEEE INFOCOM 2006 (2006)

25. Viruete, E., Ruiz, J., Fernández, J., Martínez, I.: Handbook of Research on Mobile Multimedia. In: Khalil Ibrahim, I. (ed.) Mobility Support in 4G Heterogeneous Networks for Interoperable m-Health Devices, 2nd edn. Idea Group Inc., IGI (2008) (in press)

26. Jiang, X., Xu, D.: vBET: a vm-based emulation testbed. In: Proc. of the ACM SIGCOMM workshop on Models, methods and tools for reproducible network research (MoMeTools 2003), pp. 95-104. ACM Press, New York (2003)

27. Jones, M.T.: Virtual Linux. An overview of virtualization methods, architectures, and implementations,

http: / /www-128.ibm.com/developerworks/library/

1-linuxvirt/index.html

28. Quetier, B., Neri, V., Cappello, F.: Selecting A Virtualization System For Grid/P2P Large Scale Emulation. In: Proc. of the Workshop on Experimental Grid testbeds for the assessment of large-scale distributed applications and tools (EXPGRID 2006), Paris, France (2006)

29. Van Meggelen, J., Smith, J., Madsen, L.: Asterisk, the future of telephony, 2nd edn., Cap. 12. O’Reilly, Sebastopol (2005) 